\title{
The prevalence of radiographic and MRI-defined patellofemoral osteoarthritis and structural pathology: a systematic review and meta-analysis
}

\author{
Harvi F Hart, ${ }^{1}$ Joshua I Stefanik, ${ }^{2}$ Narelle Wyndow, ${ }^{3}$ Zuzana Machotka, ${ }^{1}$ \\ Kay M Crossley ${ }^{1}$
}

\begin{abstract}
- Additional material is published online only. To view please visit the journal online (http://dx.doi.org/10.1136/ bjsports-2017-097515).

${ }^{1}$ La Trobe Sports and Exercise Medicine Research Centre, School of Allied Health, La Trobe University, Bundoora, Victoria, Australia

${ }^{2}$ Deparment of Physical Therapy, Movement and Rehabilitation Sciences, Northeastern University, Boston, Massachusetts, USA

${ }^{3}$ School of Health and

Rehabilitation Sciences, University of Queensland, Brisbane, Australia
\end{abstract}

\section{Correspondence to}

Professor Kay M Crossley, La Trobe Sport and Exercise Medicine Research Centre, School of Allied Health, College of Science, Health and Engineering, La Trobe University, Bundoora, Victoria 3086, Australia; k.crossley@latrobe. edu.au

Received 9 January 2017 Revised 23 February 2017 Accepted 27 February 2017 Published Online First 27 March 2017
ABSTRACT

Background Patellofemoral osteoarthritis (PF OA) is more prevalent than previously thought and contributes to patient's suffering from knee OA. Synthesis of prevalence data can provide estimates of the burden of PF OA.

Objective This study aims to conduct a systematic review and meta-analysis on the prevalence of PF OA and structural damage based on radiography and MRI studies in different populations.

Methods We searched six electronic databases and reference lists of relevant cross-sectional and observational studies reporting the prevalence of PF OA. Two independent reviewers appraised methodological quality. Where possible, data were pooled using the following categories: radiography and MRI studies.

Results Eighty-five studies that reported the prevalence of patellofemoral OA and structural damage were included in this systematic review. Meta-analysis revealed a high prevalence of radiographic PF OA in knee pain or symptomatic knee OA $(43 \%)$, radiographic knee OA or at risk of developing OA (48\%) and radiographic and symptomatic knee OA (57\%) cohorts. The MRI-defined structural PF damage in knee pain or symptomatic population was $32 \%$ and $52 \%$ based on bone marrow lesion and cartilage defect, respectively. Conclusion One half of people with knee pain or radiographic $\mathrm{OA}$ have patellofemoral involvement. Prevalence of MRI findings was high in symptomatic and asymptomatic population. These pooled data and the variability found can provide evidence for future research addressing risk factors and treatments for PF OA. Trial registration number PROSPERO systematic review protocol (CRD42016035649).

\section{INTRODUCTION}

Knee osteoarthritis (OA) is a leading cause of pain and disability worldwide. ${ }^{1}$ The patellofemoral joint $(\mathrm{PF})$ is commonly affected in symptomatic knee $\mathrm{OA}^{2}$ and is a substantial source of symptoms associated with knee OA. ${ }^{3}$ Further to this, the PF is often affected by OA before the tibiofemoral (TF) joint and increases the risk of TF OA development and progression. ${ }^{45}$

With a recent increase in radiography and magnetic resonance imaging (MRI) based studies focused on PF joint, the evidence on the prevalence of PF OA is expanding rapidly. A 2013 narrative literature review concluded that the prevalence of radiographic PF OA in individuals' post-ACL and/ or meniscus ruptures was approximately $50 \% .^{6} \mathrm{~A}$ recent systematic review described the prevalence of radiographic PF OA in population-based and in cohorts of people with knee pain. ${ }^{7}$ A large number of studies have reported PF OA in different populations (eg, post-traumatic and healthy individuals), and knowledge of population-specific prevalence is relevant for clinicians and researchers. An updated review with inclusion of different study samples (eg, post-traumatic, occupation-based, high risk of $\mathrm{OA}$ and healthy individuals) builds considerably on the previous systematic review ${ }^{7}$ and extends our current knowledge of PF OA.

MRI is the modality of choice to assess structural damage in epidemiological studies to detect early and subtle features of OA (eg, abnormal cartilage morphology and bone marrow lesions) not seen on radiography. ${ }^{8}$ Thus, the prevalence of PF structural damage using MRI may be higher than the prevalence determined by radiography. Including radiography and MRI-based studies in community and specific study, populations provide a comprehensive evaluation of the prevalence of PF OA and $\mathrm{PF}$ structural damage and extends prior reviews in this area. Thus, the objective of this study was to perform a systematic review and meta-analysis with the aim to determine the prevalence of PF OA using radiographs and MRI-defined structural PF damage in a variety of study populations.

\section{METHODS}

The study protocol was developed in consultation with guidelines provided by the Preferred Reporting Items for Systematic Reviews and Meta-analyses (PRISMA) statement. The protocol was prospectively registered on the PROSPERO International prospective register for systematic reviews website (http://www.crd.york.ac.uk/PROSPERO) (Registration no: CRD42016035649). The reporting of this study followed the PRISMA checklist.

\section{Literature search strategy}

Using guidelines provided by the Cochrane Collaboration, a comprehensive search strategy was devised from the following electronic databases with no date restrictions: (1) MEDLINE via OVID, (2) EMBASE via OVID, (3) CINAHL via EBSCO, (4) Scopus, (5) Web of Science and (6) SPORTDiscus. The primary search strategy included search for original publications. The search strategy was deliberately simplified to ensure inclusion of all relevant papers, with all terms searched as free text and key words (where applicable): Concept 1, Patellofemoral
Stefanik JJ, Wyndow N

et al. Br J Sports Med 
(Patello-femoral, PF, PFJ, knee joint); Concept 2, Osteoarthritis (OA, arthritis, degenerative arthritis, bone marrow lesion); and Concept 3, Prevalence (prevalence, morbidity, epidemiology, diagnosis, incidence). All search terms were exploded and scope notes from each database were examined for other possible terms for modification of search strategies. The MEDLINE search strategy was adapted for other databases (online supplementary file table 1). The search strategy was limited to English language and full text. All potential references were imported into Endnote X7 (Thomson Reuters, Carlsbad, California, USA) and duplicates were removed. Two reviewers (HFH, NW) reviewed all titles returned by the database searches and retrieved suitable abstracts. Where abstracts suggested that papers were potentially suitable, the full-text versions were screened and included in the review if they fulfilled the selection criteria. Reference lists of all publications considered for inclusion were hand searched recursively and citation tracking was completed using Google Scholar until no additional eligible publications were identified. A third reviewer was consulted in case of disagreements (JJS).

\section{Selection criteria}

Cross-sectional and longitudinal studies reporting the prevalence or frequency of PF OA or PF structural damage were included. No restrictions were placed on age, sex or method of recruitment. Reviews, case reports and unpublished studies, as well as non-human studies were excluded.

\section{Assessment of methodological quality and risk of bias}

Two independent reviewers (NW and ZM), who remained blind to authors, affiliations and the publishing journal, rated the methodological quality of included studies using the Critical Appraisal tool. ${ }^{9}$ The Critical Appraisal tool was developed to appraise prevalence and incidence-based studies and consists of eight items (maximum score possible 8). Final study ratings for each reviewer were collated and examined for discrepancies. Any inter-rater disagreement was discussed in a consensus meeting, and unresolved items were taken to a third reviewer (HFH) for consensus. Total scores were normalised to a scale ranging from 0 to 2, for each study to assign level of methodological quality. Studies were then classified as high quality $(\geq 1.4)$, moderate quality $(1.1-1.4)$ or poor quality $(<1.1)$ based on normalised scores. ${ }^{10}$

\section{Data management and statistical analysis}

For the purposes of this systematic review, we defined prevalence as the prevalence of PF OA in community-based studies and the reported frequencies of PF OA in other populations. Data pertaining to population, sample size, sex, age, type of imaging (MRI, radiography), grading criteria, units of analysis (number of participants affected or number of knees affected) and prevalence of radiographic PF OA and MRI-defined PF structural damage (isolated PF OA/PF structural damage; combined PF OA and TF OA/PF and TF structural damage; and unclear, not clearly described whether the prevalence was isolated or combined) were independently extracted and entered into an Excel spreadsheet. If sufficient data were not reported in the published article or supplementary material provided, the corresponding author was contacted to request further data. If multiple studies presented data from one cohort, the study with the most complete data was included. PF OA and MRI-defined PF structural damage prevalence data were reported for: (1) isolated, (2) combined (PF and TF) and (3) any (isolated, combined and unclear). Meta-analysis for proportions with random effects model were performed using MedCalc for Windows, V.16.8. Heterogeneity tests were also conducted and interpreted as follows: $\mathrm{I}^{2} \leq 25 \%$, low heterogeneity; $\mathrm{I}^{2}=25$ to $\leq 50 \%$, moderate heterogeneity; and $\mathrm{I}^{2} \geq 75 \%$, high heterogeneity. ${ }^{11}$ Data were divided into two categories based on imaging technique used: (1) radiography and (2) MRI.

\section{Radiography studies}

The Kellgren and Lawrence (KL) grading criteria ${ }^{12}$ and Osteoarthritis Research Society International (OARSI) atlas ${ }^{13}$ are used to define radiographic OA in the TF compartments. There is no KL or OARSI atlas definition of PF OA based on radiographs; however, both criteria are often used to quantify the severity of radiographic OA in the PF using the skyline and/or lateral radiography views. For the purposes of this systematic review and meta-analysis, osteophytes and joint space narrowing were used to define PF OA. If prevalence for multiple radiographic OA features (eg, prevalence based on osteophytes and joint space narrowing) was reported, then prevalence based on osteophytes was chosen. Data were pooled based on the following study populations: (1) community-based (individuals randomly recruited from community), (2) knee pain/ symptomatic (individuals recruited based on knee-related symptoms), (3) radiographic and symptomatic $\mathrm{OA}$ (individuals recruited based on symptoms and radiographic OA), (4) healthy individuals (no pain, injury or $\mathrm{OA}),(5)$ radiographic or high risk of OA (individuals recruited based on radiographic OA or risk of developing radiographic OA without regard to knee pain/symptoms), (6) occupational-based (individuals recruited based on their occupation/sports) and (7) post-traumatic (individuals with previous knee-related trauma, such as ACL injury or reconstruction or meniscal injury). Given that individuals recruited based on high risk of OA may or may not have had previous trauma; data from individuals with high risk of $\mathrm{OA}$ were not included in the post-traumatic category. The occupation-based category included different sporting and occupational activities such as long distance runners, shooters, graphic designers and monks. To determine the prevalence in individuals exposed to different activities, the data from sports and occupational activities were pooled together. Data were stratified based on intensity of activity (eg, high: soccer graphic and low: graphic designers) activities. For longitudinal studies, data from the latest time point (rather than baseline) were included. Within the eight study population categories, sensitivity analyses were conducted when $>1$ study reported sufficient data for pooling based on disease severity, compartment-specific OA pattern, age and sex. Disease severity was defined as mild, presence of at least mild radiographic PF OA; and definite, presence of definite radiographic PF OA (online supplementary file table 2). Compartment-specific OA pattern was defined as: (1) isolated PF OA, (2) combined PF OA and TF OA and (3) any PF OA. Age groups for sensitivity analyses were categorised as: (1) mean age: $<50$ years, (2) mean age: $\geq 50$ years. These sensitivity analyses are presented in text for any PF OA and in supplementary material (online supplementary file table 3 for the isolated and combined TF OA and PF OA groups. Where possible, medial and lateral PF OA prevalence was described.

\section{MRI studies}

Currently, there is no accepted definition of MRI-defined PF OA. A definition was proposed by Hunter $e t a l^{14}$ which included a definite osteophyte and partial or full thickness cartilage loss. However, this proposed definition of MRI-defined PF OA has not been further validated. Furthermore, most previous studies do not provide data on osteophytes to enable calculation of PF 
OA prevalence using this definition. Therefore, for the purposes of this systematic reviewwe will report MRI-defined structural damage. Data were pooled based on study populations described above (except for occupational-based population) as well as general population (studies that could not be categorised into one of the categories described above). Within each study population category, data were pooled based on cartilage defect and bone marrow lesions (BML) MRI features. Authors used the following terms to define cartilage defect: cartilage abnormalities, cartilage defect, full cartilage thickness loss, cartilage pathology and cartilage lesion; and the following terms were used to define BML, marrow abnormalities, marrow lesion and bone marrow oedema. To allow data pooling where possible other scoring systems were compared with the Whole-Organ MRI Score (WORMS) ${ }^{15}$ and MRI Osteoarthritis Knee Score (MOAKS) ${ }^{16}$ based on the explanation of the scoring system provided in the paper. Data were stratified based on compartment-specific OA pattern (isolated PF OA, combined PF OA and TF OA and any PF OA). Where possible, stratified analyses were conducted based on age (mean age: $<50$ years, $\geq 50$ years) and sex. If possible, medial and lateral PF OA prevalence was described. Most longitudinal MRI studies provided most complete data at baseline rather than at later time points (dropouts or only ORs data for later time points); thus, this review included baseline data.

\section{RESULTS}

\section{Search strategy, methodological quality and risk of bias}

The comprehensive search strategy identified 2681 titles, with the last search conducted on 25 February 2016. Following removal of duplicate publications and conference proceedings, titles of 1105 publications were evaluated. Thirteen titles were obtained from other resources (Google Scholar and hand searching). The full texts of 144 articles were retrieved, with 117 articles meeting the selection criteria. Following removal of studies with duplicate data, 85 studies (63 radiography studies, ${ }^{217-78} 24 \mathrm{MRI}$ studies $^{3979-101}$ ) were included in this systematic review (tables 1 and 2, figure 1). There was one study that reported data on radiographic PF OA and MRI-defined PF structural damage. ${ }^{35}$ The methodological quality scores ranged from 0 to 2 (out of 2) (online supplementary file table 4). There were 15 studies of high quality, 16 were moderate and 54 were low quality. Most studies scored negatively on items 1 (ie, study design/sampling method) and 6 (ie, response rate) and positively on items 4 (ie, measurement criteria) and 8 (ie, study subjects described) of the critical appraisal tool. A high level of heterogeneity was noted within radiography and MRI studies ( $\mathrm{I}^{2}$ range 96\%-100\%). The level of heterogeneity remained high $\left(\mathrm{I}^{2}\right.$ range $\left.70 \%-100 \%\right)$ when studies were further subgrouped based on population, OA severity pattern, age and sex. Exclusion of low methodological quality studies did not decrease the heterogeneity levels.

\section{Prevalence of patellofemoral OA based on radiography} Community-based population

In community-based populations, the overall prevalence of isolated PF OA from four studies ${ }^{19} 242628$ was (mean proportion: $(95 \% \mathrm{CI})) 7 \%$ (5 to 10 ), combined PF OA and TF OA from four studies ${ }^{19} 242628$ was $17 \%$ (10 to 26), and any PF OA based on nine studies ${ }^{18} 1924262839465475$ was 38\% (28 to 50) (figure 2A-C). In the any PF OA group, the prevalence of mild OA severity was 33\% (17 to 51) from three studies ${ }^{28} 4675$ and definite OA severity was 40\% (28 to 53) from six studies. ${ }^{18} 1924$ 263954 The prevalence of any PF OA in community-based population was $32 \%$ ( 24 to 42 ) in those aged 50 years or over from eight studies. ${ }^{19} 24262839465475$ Only one study described prevalence of isolated compartment-specific $\mathrm{PF} \mathrm{OA},{ }^{26}$ with prevalence of medial PF OA at $0.3 \%$ in women and $0.7 \%$ in men, and the prevalence of lateral PF OA at $1.6 \%$ in women and $3.7 \%$ in men. Sensitivity analyses based on sex revealed that the prevalence of any PF OA in women was 41\% (31 to 51) from six studies ${ }^{18} 19$ 24262875 and 47\% (23 to 71) in men from four studies. ${ }^{1819} 2426$

\section{Knee pain or symptomatic population}

Overall prevalence of isolated PF OA was 19\% (11 to 29) from eight studies, 21324252535969 combined PF OA and TF OA was $34 \%$ (25 to 43) from seven studies 2324252535969 and any PF OA was 43\% (32 to 55) from 12 studies $^{2} 212232425253596064$ 6977 (figure 2D-F). For any PF OA, the prevalence of mild and definite OA severity was 37\% (24 to 51) from seven studies 2122 32526069 and 49\% (30 to 67) from six studies, ${ }^{24253596477}$ respectively. Age-based prevalence of any PF OA in individuals under 50 years was 54\% (16 to 90) from two studies ${ }^{69} 77$ and in those 50 years or over was 43\% (31 to 56) from eight studies. 212242 53596064 Sex-based prevalence of any PF OA was 46\% (23 to 70) in women ${ }^{5360}$ and 58\% (27 to 86) in men. ${ }^{536069}$

\section{Radiographic and symptomatic knee osteoarthritis}

Overall prevalence of isolated PF OA was 20\% (11 to 32) from four studies, 25303355 combined PF OA and TF OA was 43\% (8 to 83 ) from two studies ${ }^{25} 55$ and any PF OA was 57\% (43 to 70) from 13 studies 25273033353741455055617478 (figure 2G-I). In the any PF OA group, the prevalence of mild severity was 56\% (41 to 70 ) from 12 studies. ${ }^{25} 3033353741455055617478$ The prevalence in individuals 50 years or over was 58\% (42 to 72) from 12 studies 252730333537414555617478 and the prevalence of any PF OA in women was 36\% (33 to 38$)^{2745}$ and men was 35\% (16 to 58) from two studies. ${ }^{2745}$

\section{Healthy individuals}

Data from four studies were included in meta-analyses to determine the prevalence of PF OA in healthy individuals. ${ }^{34} 606372$ Overall prevalence of any PF OA in healthy individuals (no pain, injury or OA) was $17 \%$ (6 to 33) (figure 3A). Sensitivity analyses based on sex could only be performed in women revealing the prevalence of PF OA in healthy women at 15\% (1 to 43) from two studies. ${ }^{6072}$

\section{Radiographic knee OA or at risk of developing OA}

Overall prevalence of any PF OA in individuals with radiographic OA or at risk of OA was 48\% (35 to 61) from four studies ${ }^{36} 38$ 4348 (figure 3B), with prevalence based on mild and definite OA severity as follows: 54\% (17 to 89 ) from two studies ${ }^{36} 48$ and $45 \%$ (30 to 60 ) from two studies, ${ }^{38} 43$ respectively. In this group, the prevalence of any PF OA in women was 41\% (8 to 80) from two studies. ${ }^{3843}$

\section{Occupation-based population}

Four studies reported occupation-based prevalence of PF OA. ${ }^{51687276}$ Overall prevalence of any PF OA in individuals in occupations or sports such as long distance running, soccer, shooting, floor layers, graphic designers and monks was $21 \%$ (9 to 37) (figure 3C). For any PF OA, the prevalence based on mild OA severity was 29\% (10 to 52) from three studies. ${ }^{687276}$ The prevalence of any PF OA in individuals 50 years and over was $18 \%$ (9 to 28) from three studies. ${ }^{5168} 72$ Sensitivity analyses based on sex revealed the prevalence of any PF OA in men was $14 \%$ (9 to 20) from two studies. ${ }^{5168}$ Analysis could not be 


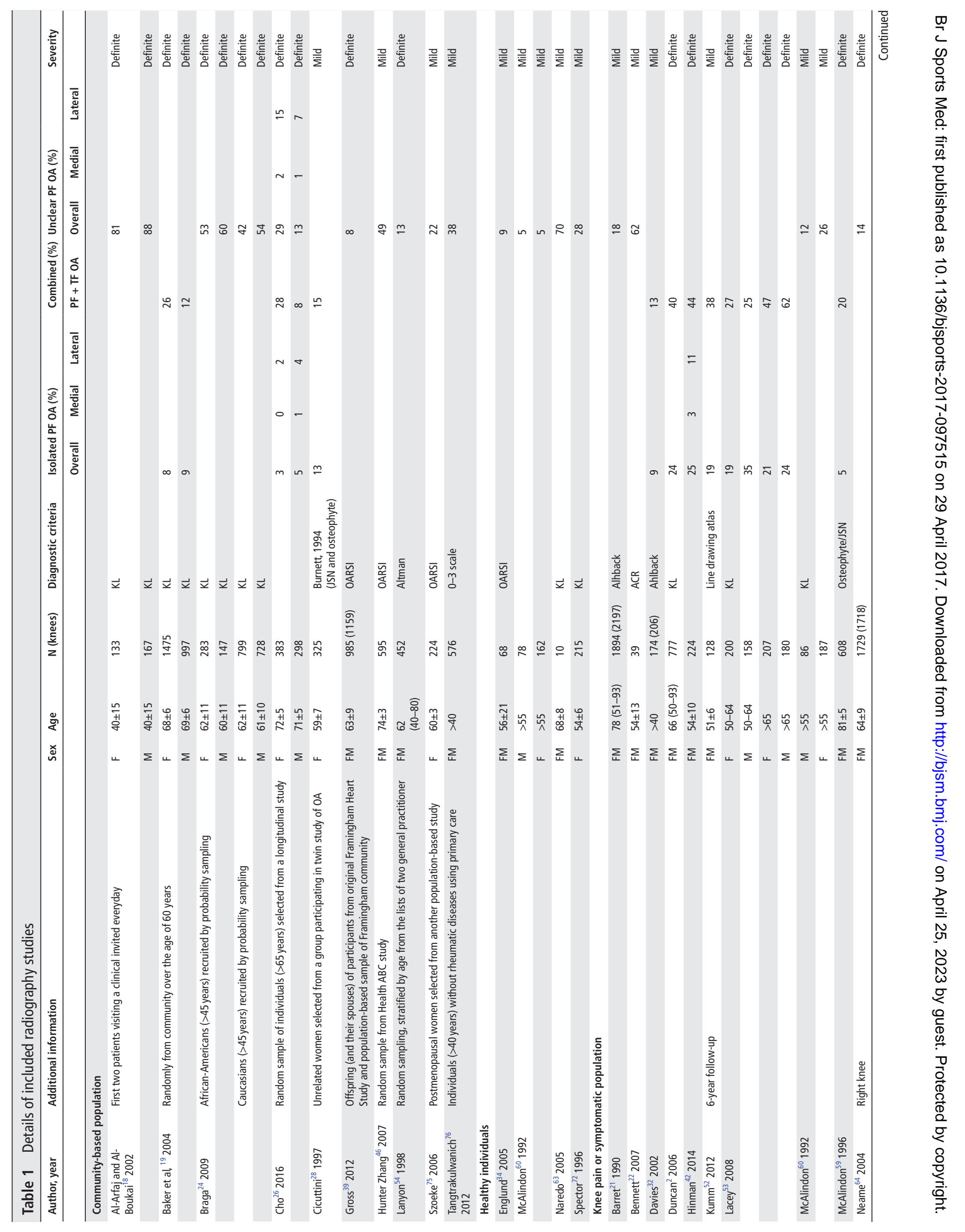




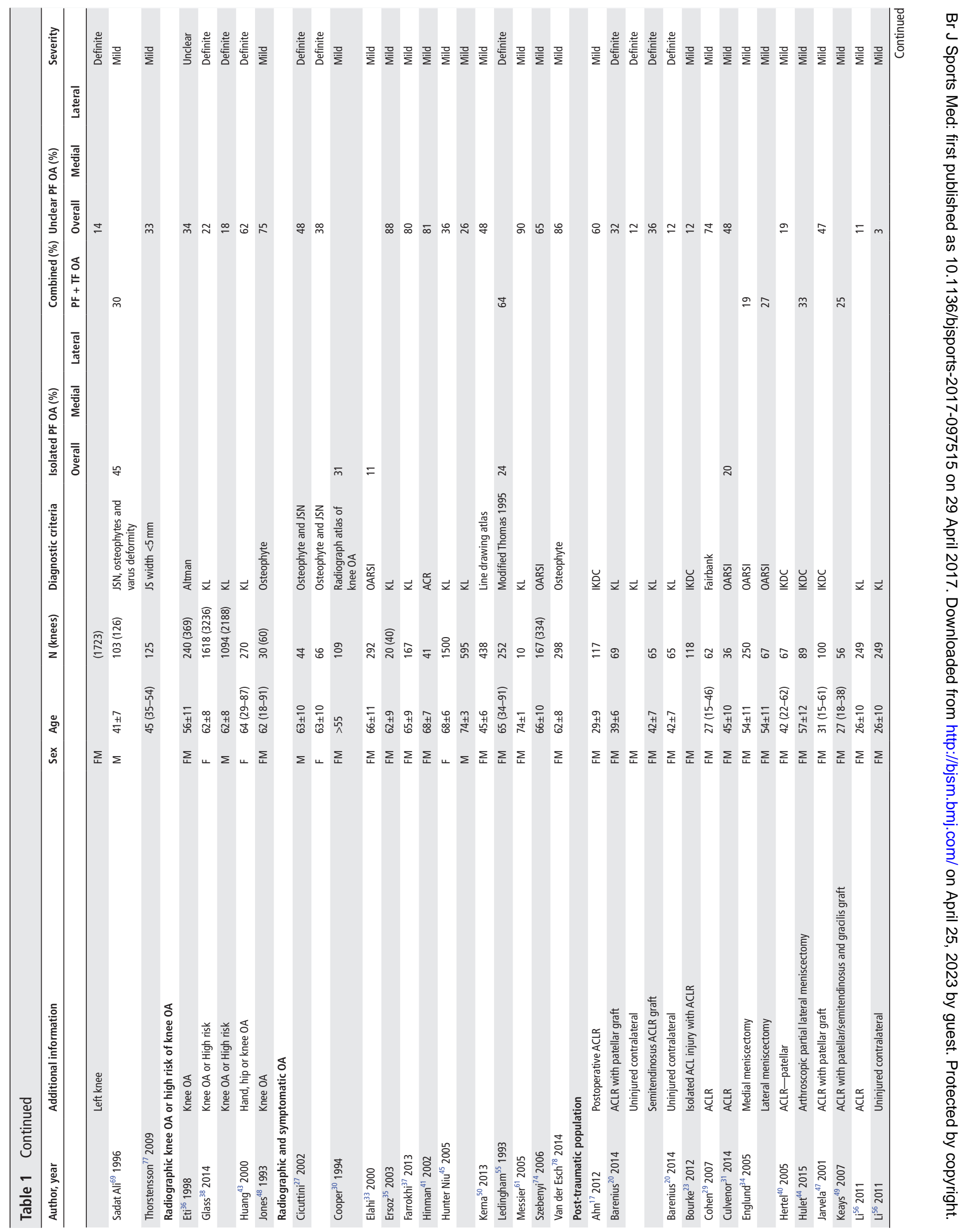




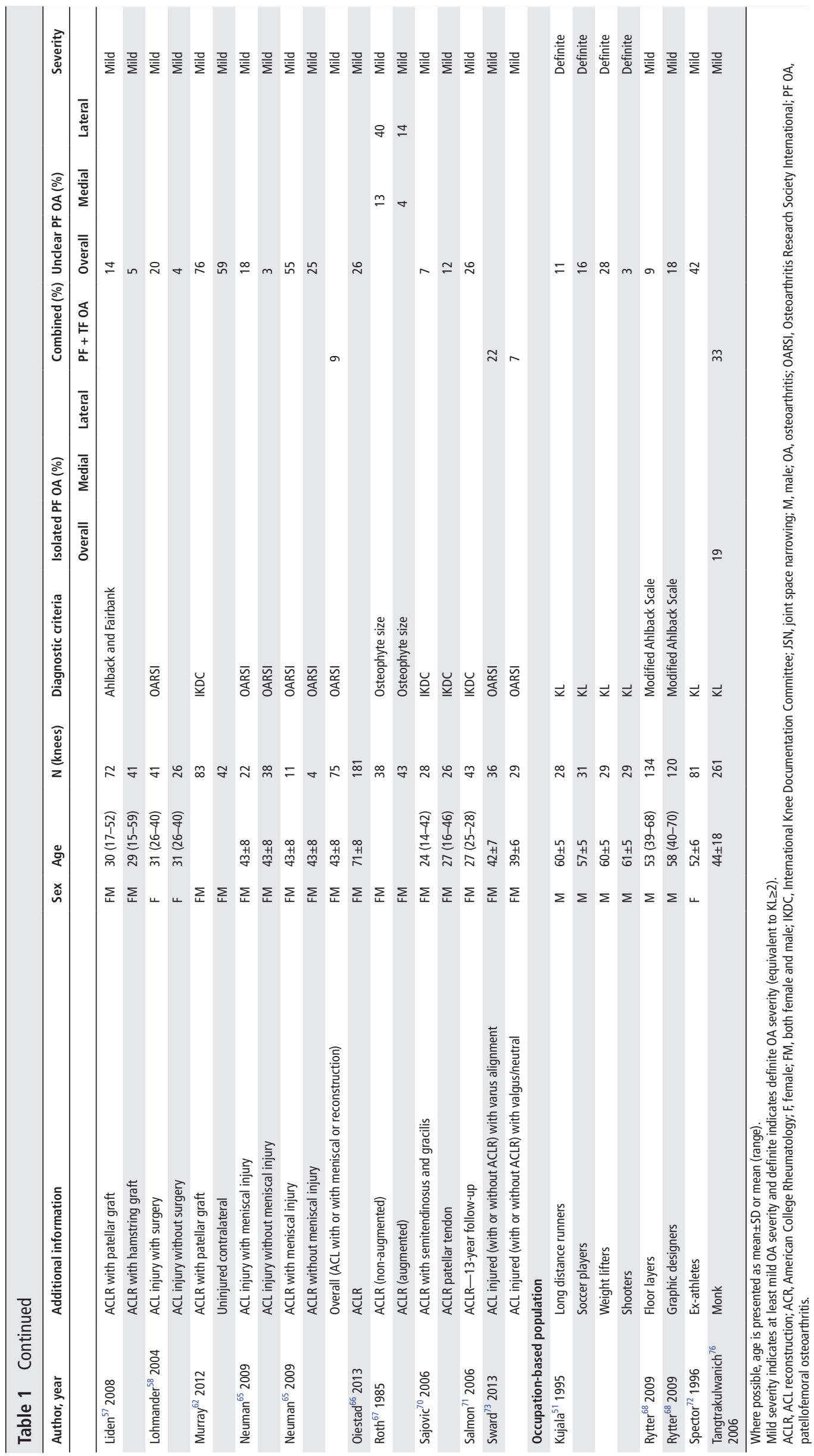




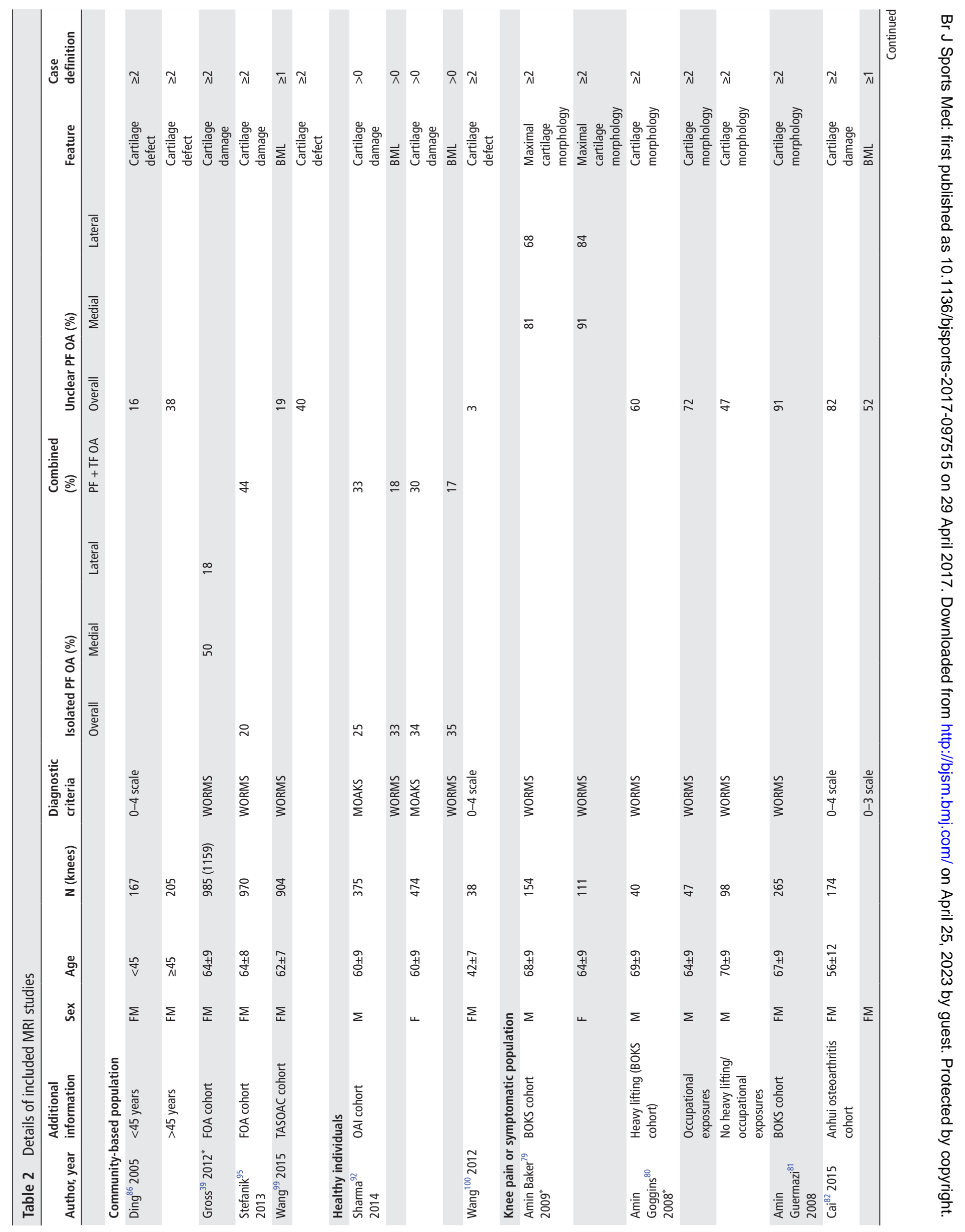




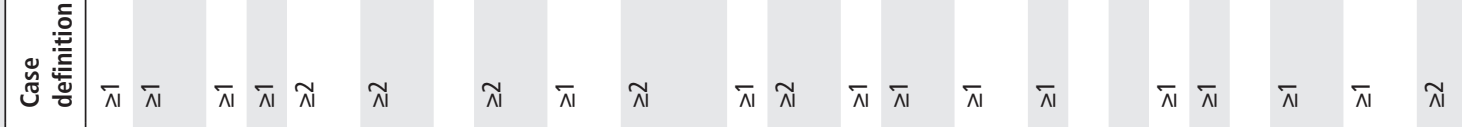

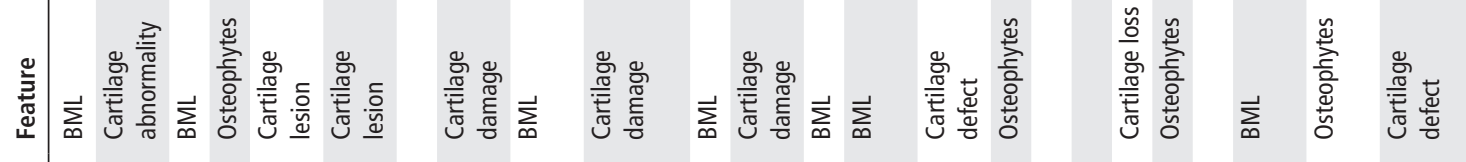
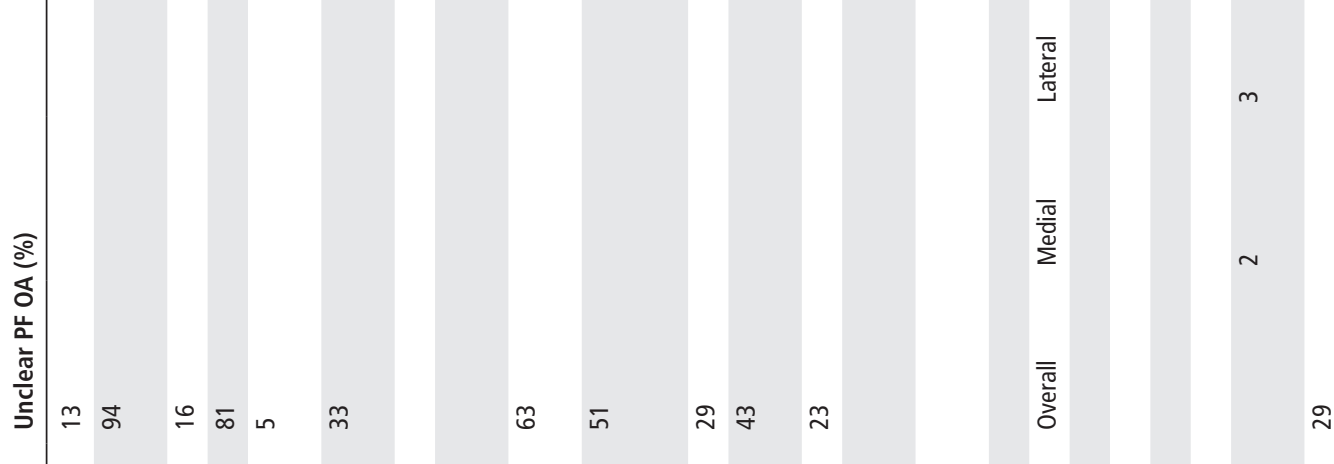

हृँ

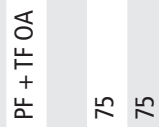

m $m$

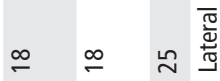

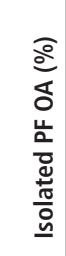

8 ก

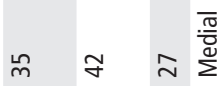

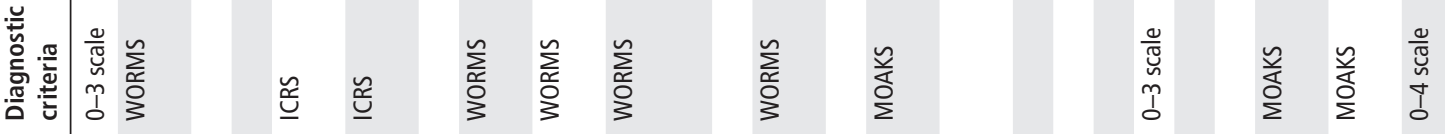

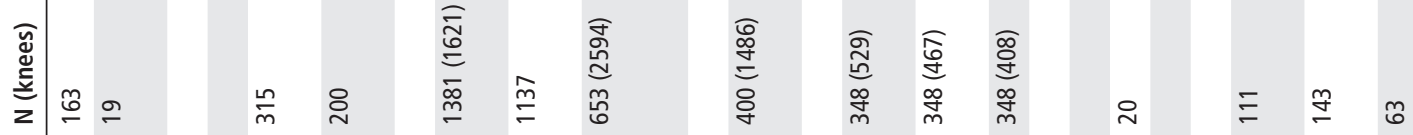

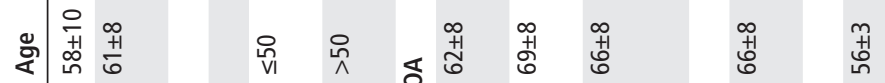

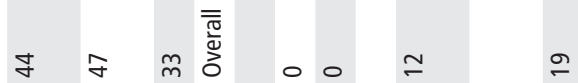

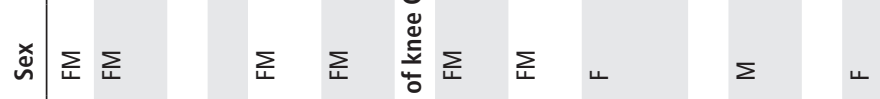

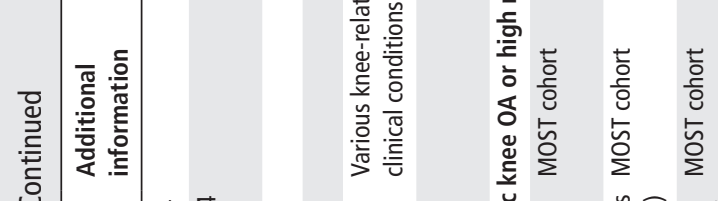

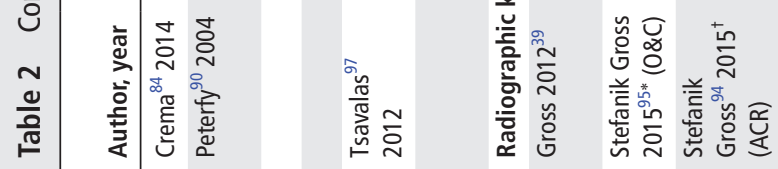

In

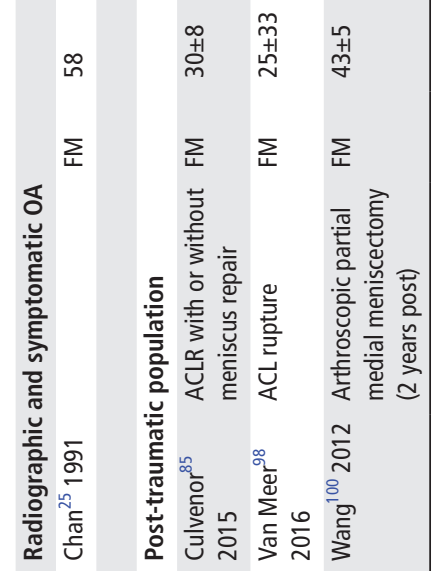

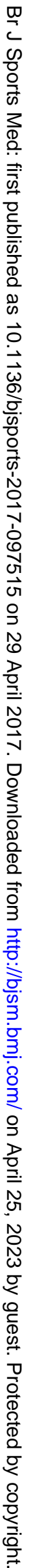




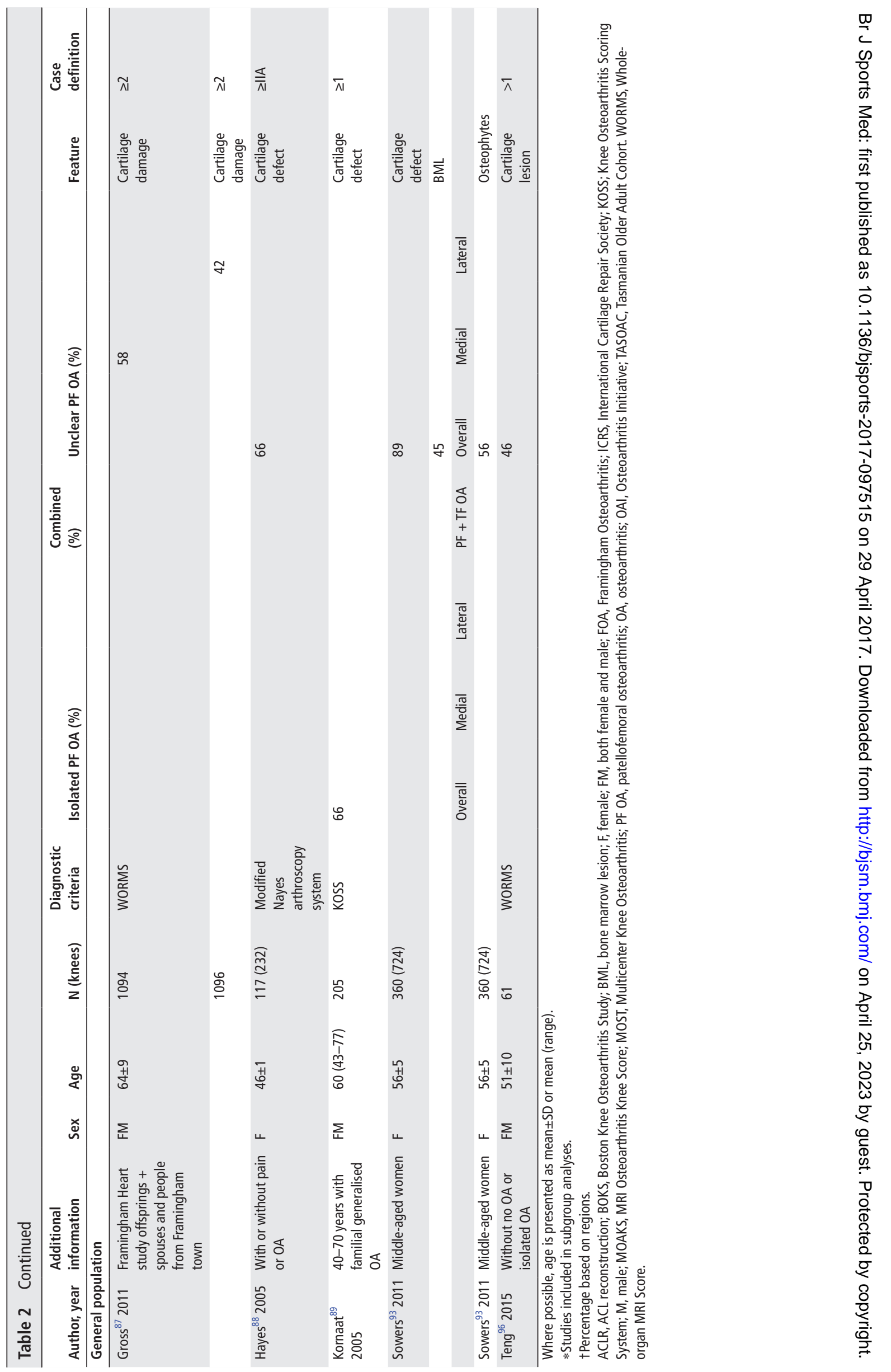




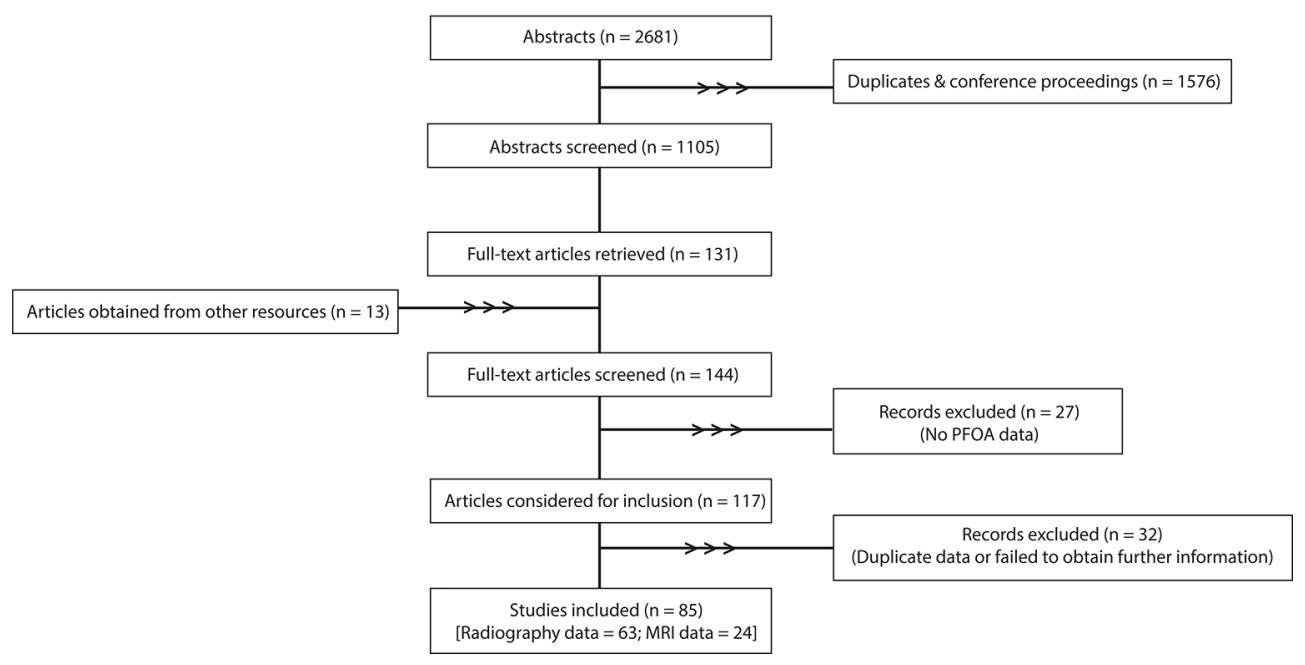

Figure 1 Flow chart of the study selection process. PFOA, patellofemoral osteoarthritis.

performed in women. For any PF OA, the prevalence of any PF OA in high-intensity activity population was $19 \%$ (11 to 29 ) from one study ${ }^{51}$ and $19 \%$ ( 3 to 45 ) in low-intensity activity population based on three studies. 516876

\section{Post-traumatic population}

The overall prevalence of isolated PF OA from two studies was $17 \%$ (5 to 34 ) from two studies ${ }^{49} 65$ (figure 3D). In the injured knee, the overall of prevalence of any PF OA in post-traumatic population (range: 5 to 22 years) was 27\% (19 to 34) from

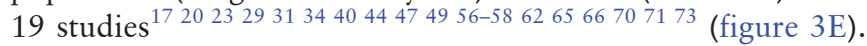
For any PF OA, the prevalence of mild OA severity was $26 \%$ (18 to 34) from 18 studies. ${ }^{17232931344044474956-586265667071}$ ${ }^{73}$ Sensitivity analyses based on age revealed the prevalence of any PF OA was 27\% (18 to 36 ) in individuals under 50 years ${ }^{17}$ $2023293140474956-586566707173$ and 26\% (17 to 35) in those 50 years or over. ${ }^{3444}$ In the uninjured knee, overall prevalence of any PF OA was 18\% (3 to 42) from three studies, ${ }^{20} 5662$ with

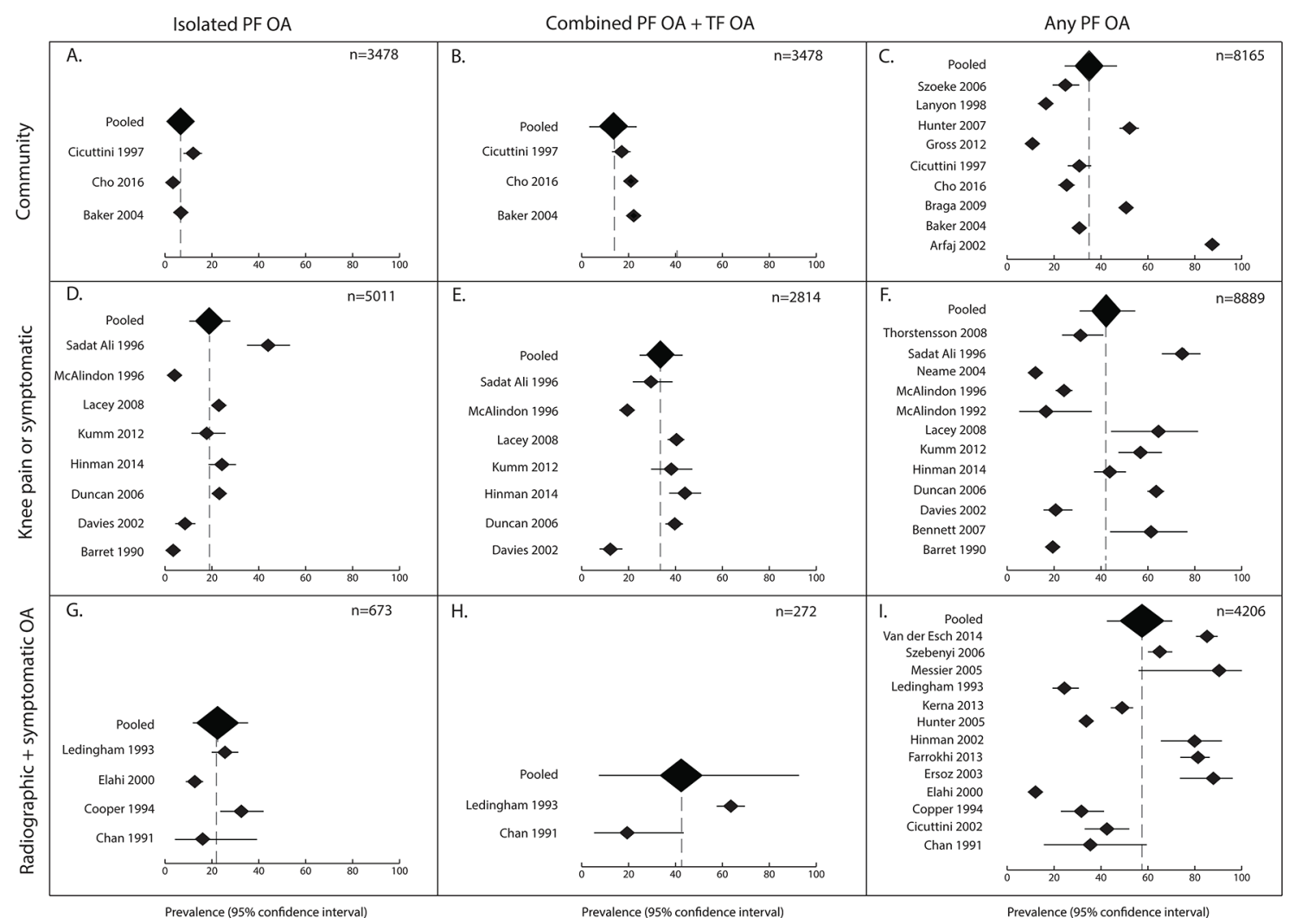

Figure 2 Prevalence of patellofemoral osteoarthrits (PF OA) in community, knee pain or symptomatic and radiographic and symptomatic OA populations. TF, tibiofemoral. 


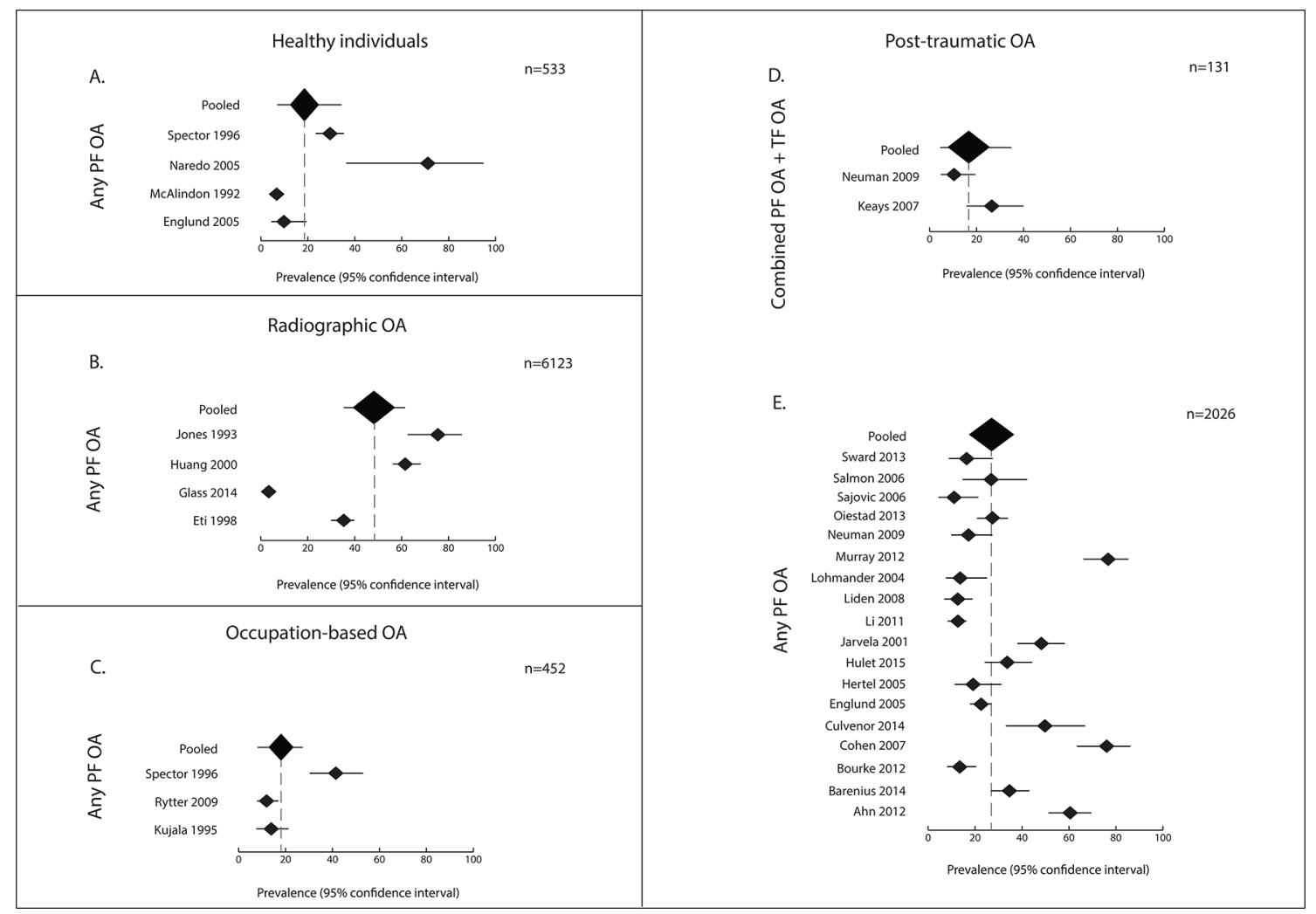

Figure 3 Prevalence of PF OA in healthy individuals, radiographic OA, occupation-based OA and post-traumatic OA populations. PF OA, patellofemoral osteoarthritis; TF, tibiofemoral.

prevalence of mild OA severity at 25\% (2 to 87 ) from two studies. $^{5662}$

\section{Prevalence of PF OA based on MRI \\ Community-based population}

The prevalence of isolated PF structural damage and combined $\mathrm{PF}$ and TF structural damage based on cartilage defect were $20 \%$ and $44 \%$ (respectively) and BML was $18 \%$ and 22\% (respectively) based on a single study. ${ }^{95}$ The prevalence of any PF structural damage based on cartilage defects was 44\% (25 to 65) from three studies ${ }^{869599}$ and BML was 29\% (11 to 51) from two studies $^{9599}$ (figure 4A-B).

\section{Knee pain or symptomatic population}

The prevalence of overall isolated PF structural damage and combined PF and TF structural damage could not be calculated for this study population. The prevalence of any PF structural damage was 52\% (9 to 93) based on cartilage defect ${ }^{818297}$ and $32 \%$ (3 to 72 ) based on $\mathrm{BML}^{82} 84$ (figure 4C, D). Data from one study could not be pooled because of WORMS definition used for OA diagnosis (cartilage damage defined as $\geq 1$ grade in this study compared with $\geq 2$ grade used in other studies), ${ }^{90}$ with PF structural damage prevalence of $94 \%, 16 \%$ and $81 \%$ based on cartilage defect, BML and osteophytes, respectively. Data stratified based on age revealed that the prevalence of any PF structural damage was $71 \%$ (33 to 97) in individuals 50 years or over based on cartilage defect. ${ }^{81} 8297$

\section{Radiographic knee osteoarthritis or at risk of developing OA}

An overall prevalence of isolated PF structural damage, combined PF and TF structural damage and any PF structural damage based on cartilage defect or BML could not be determined for this study population. Two studies reported prevalence of isolated PF structural damage in the medial and lateral PF compartments based on cartilage defect ${ }^{39} 91$ and BML. ${ }^{39} 101$ The prevalence of isolated medial and lateral PF structural damage was 56\% (29 to 81 ) and $27 \%$ (11 to 46 ), respectively, ${ }^{39} 91$ based on cartilage defect and 28\% (17 to 41) and 15\% (11 to 20), respectively, ${ }^{39} 101$ based on BML (figure 4E,F). A single study described PF structural damage prevalence based on PF compartment regions (not based on number of individuals or knees) ${ }^{94}$ and reported prevalence of any PF structural damage based on cartilage defect and BML in women (51\% and 29\%, respectively) and men (43\% and $23 \%$, respectively). ${ }^{94}$ No further analyses could be conducted in this study population.

\section{Healthy individuals}

The overall prevalence of any PF structural damage based on cartilage defect was 40\% (19 to 63) ${ }^{92100}$ (figure 4G). Since there were only two studies included in this study population, no further analyses could be conducted.

\section{Radiographic and symptomatic knee OA}

The prevalence of combined PF and TF structural damage was $75 \%$ based on cartilage defect and osteophytes from a single study, ${ }^{83}$ and no further analyses could be conducted.

\section{Post-traumatic population}

Two studies reported prevalence based on osteophytes in ACL injured or reconstructed, ${ }^{8598}$ with the prevalence of any PF structural damage at $29 \%{ }^{98}$ and compartment-specific prevalence of medial and lateral PF structural damage at 23\% and $7 \%$, respectively. ${ }^{85}$ The prevalence of medial and lateral PF structural damage based on BML were 2\% and 3\%, respectively. ${ }^{85}$ The 


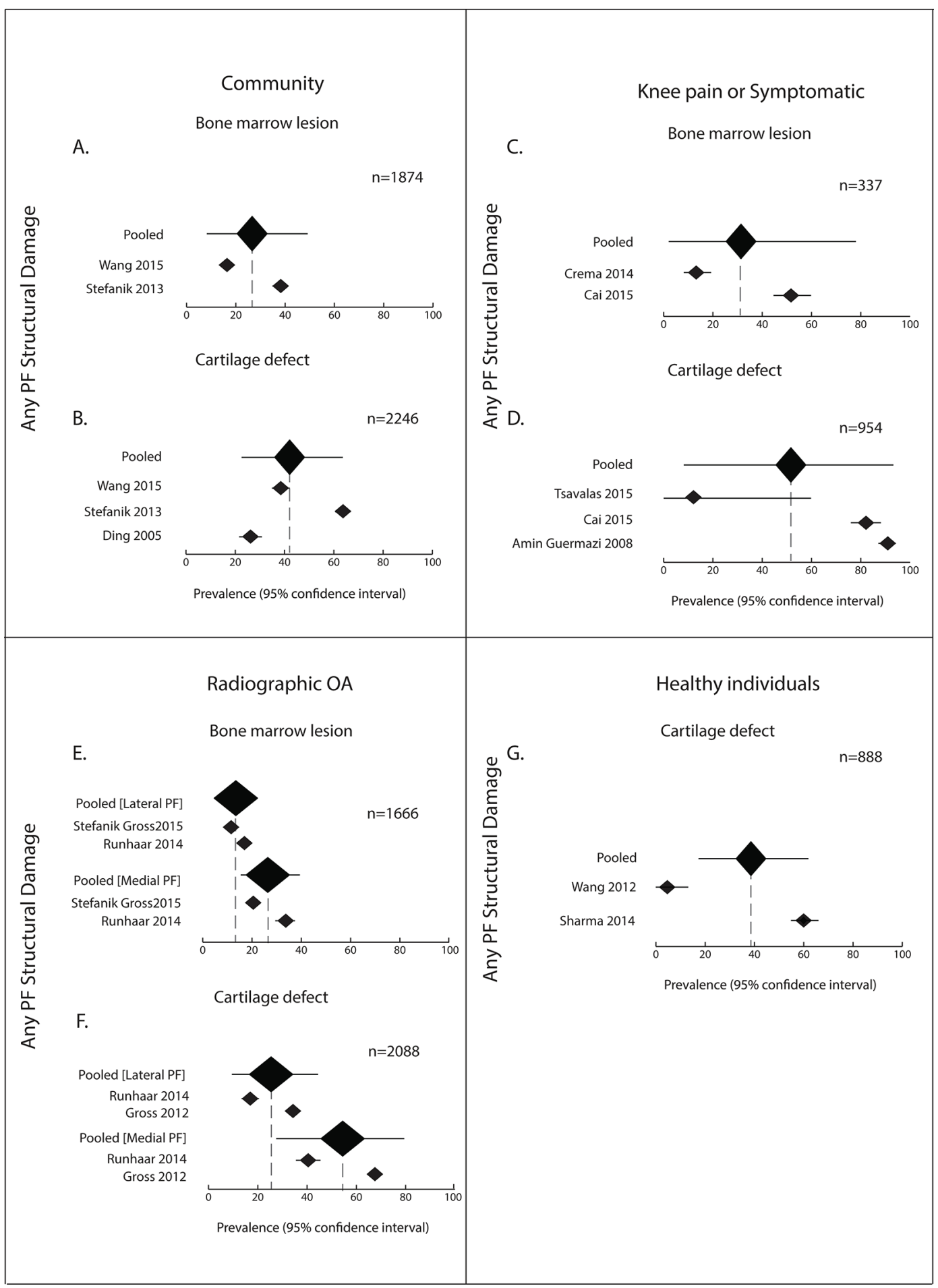

Figure 4 Prevalence of MRI-defined PF structural damage in community, knee pain or symptomatic, radiographic OA and healthy individual populations. PF OA, patellofemoral osteoarthritis.

prevalence of any PF structural damage was $36 \%$ in an ACL ruptured population based on cartilage defect. ${ }^{98}$ In individuals 2 years post arthroscopic partial medial meniscectomy, the prevalence of isolated PF structural damage was 19\% based on cartilage defect. ${ }^{100}$

\section{General population}

Five studies were included in the general population category. ${ }^{87-899396}$ The cartilage defect based prevalence of any PF structural damage was 49\% (36 to 62) from two studies using the WORMS ${ }^{8796}$ and was 75\% (56 to 91) from three studies using the KOSS. ${ }^{88} 8993$ The prevalence of any PF structural damage based on BML and osteophytes were $45 \%$ and $56 \%$, respectively. ${ }^{93}$

\section{DISCUSSION}

\section{Summary of findings}

This systematic review with meta-analysis synthesised prevalence of PF OA and included 85 studies. Meta-analysis revealed 
the prevalence of any radiographic PF OA in knee pain or symptomatic, radiographic TF OA or at risk of developing TF OA, and radiographic and symptomatic knee OA cohorts was $43 \%$, $48 \%$ and $57 \%$, respectively. The prevalence of any MRI-defined PF structural damage in knee pain or symptomatic population was $32 \%$ and $52 \%$ based on BML and cartilage defect, respectively. This systematic review and meta-analysis highlights the high prevalence of $\mathrm{PF} \mathrm{OA} / \mathrm{PF}$ structural damage in a wide range of study populations using different imaging tools.

One half $(43 \%-57 \%)$ of people with symptoms and/or established radiographic TF OA had PF OA based on radiography. Similarly, a high prevalence of post-traumatic population exhibited signs of PF OA ( 30). With such a high prevalence of PF OA, treatments designed specifically for the PF compartment may be required in the OA management strategy. ${ }^{102}{ }^{103}$ Clinicans should assess for symptoms of PF pain or PF OA and treat patients accordingly. The few studies that specifically evaluated interventions such as exercise, physical therapy, taping and bracing to address PF OA 9899104 provide some evidence for their use. While some studies hypothesise that there is a potential continuum of PF pain to PF $\mathrm{OA}^{105}$; no high-quality evidence has supported the association between PF pain in younger individuals to the development of PF OA. ${ }^{106}$ Unfortunately, studies included in the knee pain or symptomatic OA population category did not differentiate between PF pain and generalised knee pain. Therefore, in the current systematic review, we were not able to determine the prevalence of PF OA in a PF pain population.

Healthy and community cohorts are also likely to demonstrate some PF OA, with radiographic PF OA evident in $17 \%$ and $38 \%$, respectively. Since most studies in the community-based meta-analysis were conducted in individuals over the age of 50 years, it appears that radiographic PF OA may be a natural accompaniment to ageing. The only study with a mean age of $\leq 50$ years (but a large range 20 to 93 years), described a particularly high PF OA prevalence in women (81\%) and men (88\%). The authors hypothesised that cultural factors in Saudi Arabia, such as sitting cross-legged, squatting and praying with knees fully flexed on the ground, may contribute to the high prevalance. Exclusion of this data from meta-analysis revealed the prevalence of any PF OA was $32 \%$ in the community population.

The prevalence of MRI-defined PF structural damage in knee pain or symptomatic population was $52 \%$, which was similar to the healthy (40\%), community (44\%) and general population (49\%) cohorts. The high prevalence of MRI-defined PF structural damage may reflect the ability of MRI to detect early changes in the joint that are not visible on radiographs. However, it is unclear whether these findings represent PF OA, as there is no accepted and validated MRI definition of OA. MRI features such as cartilage damage and BMLs can predict incident radiographic $\mathrm{OA},{ }^{107}$ development of knee pain ${ }^{108}$ and future total knee replacement. ${ }^{109}$ Thus, it is plausible that these MRI findings may represent early stages of the PF OA disease process. Further research is needed to investigate the clinical relevance of MRI-defined PF structural damage.

The current systematic review extends on the results from a prior study. ${ }^{7}$ The previous systematic review reported the radiographic prevalence of $\mathrm{PF} \mathrm{OA}$ in population-based and symptom- based population; whereas, the current review reported prevalence of PF OA in multiple different populations. Thus, an additional 32 studies were included in the meta-analysis. Further to this, the current review included meta-analysis on prevalence of MRI-defined PF structural damage. Furthermore, the current study extends on the findings from the earlier review by categorising data into multiple study populations and data pooling with subanalysis based on age, sex, compartment-specific OA pattern and OA severity pattern to obtain more accurate estimations of prevalence.

\section{Limitations}

This systematic review is not without limitations. First, a very high level of heterogeneity was noted, particularly in the any PF OA group. The inclusion of isolated PF OA, combined PF OA and TF OA, and unclear PF OA (isolated or combined) data in the any PF OA group may explain the high level of heterogeneity. Other potential sources of heterogeneity include differences in diagnostic criteria, populations and case definitions. Second, all relevant studies were included in this systematic review, regardless of methodological quality. Data from 54 low methodological quality studies were included in this review. While this systematic review is subject to bias through the inclusion of low-quality studies, the levels of evidence applied to the pooled data take into account quality, quantity and homogeneity of studies. Third, we restricted the search to studies published in English. Inclusion of data from non-English language studies may alter the outcomes. Fourth, a number of diagnostic criteria were converted to allow data pooling, which may have influenced the results of this systematic review. Fifth, PF structural damage based on MRI should be interpreted with some caution, as fewer studies contributed to meta-analysis within each study population. Lastly, we recognise that there is no accepted and validated definition of radiographic or MRI defined PF OA. Because of this the prevalence data will largely differ in any given study based on different definitions, which may have influenced the results.

\section{Recommendations}

While conducting this systematic review, we identified that prevalence data were not well presented in many studies. We recommend that future studies more clearly describe prevalence data based on OA patterns (eg, isolated PF OA vs combined PF OA and TF OA, medial vs lateral PF OA), OA severity (eg, none, mild and moderate) and subgroups (eg, age, sex). Further to this, discrepancies in diagnostic criteria definitions and reporting were noted; therefore, the PF OA definitions should be clearly stated. Better standardisation of data presentation in future studies will help to better understand PF OA epidemiology.

\section{Implications for research and practice}

$\mathrm{PF} \mathrm{OA}$ is an important source of symptoms in knee OA, and is strongly associated with disability. ${ }^{60}$ Our systematic review and meta-analysis revealed the prevalence of PF OA is highly based on radiography and MRI in community, symptomatic, radiographic knee OA and traumatic knee OA populations. Therefore, well-designed studies are required to evaluate biomechanical, functional and psychological impairments associated with PF OA. Addressing potentially modifiable risk factors for PF OA may reduce the risk of development and progression of PF OA and may have implications for TF disease. This systematic review also revealed a higher prevalence of combined PF OA and TF OA pattern than isolated PF OA; therefore, it is important to explore interventions that target both PF and TF joints.

\section{CONCLUSIONS}

Synthesis of prevalence data on PF OA and MRI-defined PF structural damage indicates that signs of PF damage are common and should not be ignored in research or clinical practice. In the future, MRI might become highly relevant to identify patients at 
early disease stages where the disease process may still be reversible and amenable to interventions.

\section{What are the new findings?}

- Patellofemoral OA is prevalent in individuals in a very wide range of settings - in asymptomatic individuals and in patients with knee pain.

- The prevalence rates are influenced by different diagnostic criteria.

- MRI-defined patellofemoral structural damage criteria may assist in identifying patients at early disease stages.

Contributors All authors were fully involved in the study and preparation of the manuscript. Each author has read and concurs with the content in the final manuscript. Study conception: HFH, KMC, JJS. Protocol: HFH, KMC, JJS. Search strategy: HFH, KMC, JJS. Abstract screening: HFH, NW. Data extraction: HFH, JJS, KMC. Methodological quality ratings: NW, ZM. Data analysis and interpretation: HFH, JJS, KMC. Manuscript preparation: HFH, JJS, KMC, NW, ZM.

Competing interests None declared.

Provenance and peer review Not commissioned; externally peer reviewed.

(c) Article author(s) (or their employer(s) unless otherwise stated in the text of the article) 2017. All rights reserved. No commercial use is permitted unless otherwise expressly granted.

\section{REFERENCES}

1 Cross M, Smith E, Hoy D, et al. The global burden of hip and knee osteoarthritis: estimates from the global burden of disease 2010 study. Ann Rheum Dis 2014;73:1323-30

2 Duncan RC, Hay EM, Saklatvala J, et al. Prevalence of radiographic osteoarthritis-it all depends on your point of view. Rheumatology 2006:45:757-60.

3 Duncan R, Peat G, Thomas E, et al. Does isolated patellofemoral osteoarthritis matter? Osteoarthritis Cartilage 2009;17:1151-5.

4 Duncan R, Peat G, Thomas E, et al. Incidence, progression and sequence of development of radiographic knee osteoarthritis in a symptomatic population. Ann Rheum Dis 2011;70:1944-8.

5 Stefanik JJ, Guermazi A, Roemer FW, et al. Changes in patellofemoral and tibiofemoral joint cartilage damage and bone marrow lesions over 7 years: the multicenter osteoarthritis study. Osteoarthritis Cartilage 2016;24:1160-6.

6 Culvenor AG, Cook JL, Collins NJ, et al. Is patellofemoral joint osteoarthritis an under-recognised outcome of anterior cruciate ligament reconstruction? A narrative literature review. Br I Sports Med 2013;47:66-70.

7 Kobayashi S, Pappas E, Fransen M, et al. The prevalence of patellofemoral osteoarthritis: a systematic review and meta-analysis. Osteoarthritis Cartilage 2016;24:1697-707

8 Guermazi A, Roemer FW, Burstein D, et al. Why radiography should no longer be considered a surrogate outcome measure for longitudinal assessment of cartilage in knee osteoarthritis. Arthritis Res Ther 2011;13:247.

9 Loney PL, Chambers LW, Bennett KJ, et al. Critical appraisal of the health research literature: prevalence or incidence of a health problem. Chronic Dis Can 1998:19:170-6.

10 Genaidy AM, Lemasters GK, Lockey J, et al. An epidemiological appraisal instrument - a tool for evaluation of epidemiological studies. Ergonomics 2007; 50:920-60.

11 Higgins JPT. The Cochrane Collaboration,. Cochrane handbook for systematic reviews of interventions, 2008. ISBN: 978-0-470-69951-5.

12 Kellgren JH, Lawrence JS. Radiological assessment of osteo-arthrosis. Ann Rheum Dis 1957:16:494-502.

13 Altman RD, Gold GE. Atlas of individual radiographic features in osteoarthritis, revised. Osteoarthritis Cartilage 2007;15(Suppl A):A1-A56.

14 Hunter DJ, Arden N, Conaghan PG, et al. Definition of osteoarthritis on MRI: results of a delphi exercise. Osteoarthritis Cartilage 2011;19:963-9.

15 Peterfy CG, Guermazi A, Zaim S, et al. Whole-Organ magnetic resonance imaging score (WORMS) of the knee in osteoarthritis. Osteoarthritis Cartilage 2004;12:177-90.

16 Hunter DJ, Guermazi A, Lo GH, Gh L, et al. Evolution of semi-quantitative whole joint assessment of knee OA: MOAKS (MRI osteoarthritis knee score). Osteoarthritis Cartilage 2011;19:990-1002.
17 Ahn JH, Kim JG, Wang JH, et al. Long-term results of anterior cruciate ligament reconstruction using bone-patellar tendon-bone: an analysis of the factors affecting the development of osteoarthritis. Arthroscopy 2012;28:1114-23.

18 Al-Arfaj A, Al-Boukai AA. Prevalence of radiographic knee osteoarthritis in Saudi Arabia.. Clin Rheumatol 2002;21:142-5.

19 Baker KR, Xu L, Zhang Y, et al. Quadriceps weakness and its relationship to tibiofemoral and patellofemoral knee osteoarthritis in Chinese: the Beijing osteoarthritis study. Arthritis Rheum 2004;50:1815-21.

20 Barenius B, Ponzer S, Shalabi A, et al. Increased risk of osteoarthritis after anterior cruciate ligament reconstruction: a 14-year follow-up study of a randomized controlled trial. Am J Sports Med 2014:42:1049-57.

21 Barrett JP, Rashkoff E, Sirna EC, et al. Correlation of roentgenographic patterns and clinical manifestations of symptomatic idiopathic osteoarthritis of the knee. Clin Orthop Relat Res 1990;253:179-83.

22 Bennett AN, Crossley KM, Brukner PD, et al. Predictors of symptomatic response to glucosamine in knee osteoarthritis: an exploratory study. Br J Sports Med 2007:41:415-9.

23 Bourke HE, Gordon DJ, Salmon LJ, et al. The outcome at 15 years of endoscopic anterior cruciate ligament reconstruction using hamstring tendon autograft for 'isolated' anterior cruciate ligament rupture. J Bone Joint Surg Br 2012;94:630-7.

24 Braga L, Renner JB, Schwartz TA, et al. Differences in radiographic features of knee osteoarthritis in African-Americans and caucasians: the johnston county osteoarthritis project. Osteoarthritis Cartilage 2009;17:1554-61.

25 Chan WP, Lang P, Stevens MP, et al. Osteoarthritis of the knee: comparison of radiography, $\mathrm{CT}$, and MR imaging to assess extent and severity. AJR Am J Roentgenol 1991;157:799-806.

$26 \mathrm{Cho} \mathrm{HJ,} \mathrm{Gn} \mathrm{KK,} \mathrm{Kang} \mathrm{JY,} \mathrm{et} \mathrm{al.} \mathrm{Epidemiological} \mathrm{characteristics} \mathrm{of} \mathrm{patellofemoral}$ osteoarthritis in elderly Koreans and its symptomatic contribution in knee osteoarthritis. Knee 2016;23:29-34.

27 Cicuttini F, Wluka A, Wang Y, et al. The determinants of change in patella cartilage volume in osteoarthritic knees. J Rheumatol 2002;29:2615-9.

28 Cicuttini FM, Spector T, Baker J. Risk factors for osteoarthritis in the tibiofemoral and patellofemoral joints of the knee. J Rheumatol 1997;24:1164-7.

29 Cohen M, Amaro JT, Ejnisman B, et al. Anterior cruciate ligament reconstruction after 10 to 15 years: association between meniscectomy and osteoarthrosis. Arthroscopy 2007;23:629-34

30 Cooper C, McAlindon T, Snow S, et al. Mechanical and constitutional risk factors for symptomatic knee osteoarthritis: differences between medial tibiofemoral and patellofemoral disease. J Rheumato/ 1994:21:307-13.

31 Culvenor AG, Lai CC, Gabbe BJ, et al. Patellofemoral osteoarthritis is prevalent and associated with worse symptoms and function after hamstring tendon autograft $\mathrm{ACL}$ reconstruction. Br I Sports Med 2014;48:435-9.

32 Davies AP, Vince AS, Shepstone $L$, et al. The radiologic prevalence of patellofemoral osteoarthritis. Clin Orthop Relat Res 2002:402:206-12.

33 Elahi S, Cahue S, Felson DT, et al. The association between varus-valgus alignment and patellofemoral osteoarthritis. Arthritis Rheum 2000:43:1874-80.

34 Englund M, Lohmander LS. Patellofemoral osteoarthritis coexistent with tibiofemoral osteoarthritis in a meniscectomy population. Ann Rheum Dis 2005;64:1721-6.

35 Ersoz M, Ergun S. Relationship between knee range of motion and KellgrenLawrence radiographic scores in knee osteoarthritis. Am J Phys Med Rehabil 2003;82:110-5.

36 Eti E, Kouakou HB, Daboiko JC, et al. Epidemiology and features of knee osteoarthritis in the ivory coast. Rev Rhum Engl Ed 1998;65:766-70.

37 Farrokhi S, Piva SR, Gil AB, et al. Association of severity of coexisting patellofemoral disease with increased impairments and functional limitations in patients with knee osteoarthritis. Arthritis Care Res 2013;65:544-51.

38 Glass N, Segal NA, Sluka KA, et al. Examining sex differences in knee pain: the multicenter osteoarthritis study. Osteoarthritis Cartilage 2014;22:1100-6.

39 Gross KD, Niu J, Stefanik JJ, et al. Breaking the law of Valgus: the surprising and unexplained prevalence of medial patellofemoral cartilage damage. Ann Rheum Dis 2012;71:1827-32.

40 Hertel P, Behrend H, Cierpinski T, et al. ACL reconstruction using bone-patellar tendon-bone press-fit fixation: 10-year clinical results. Knee Surg Sports Traumatol Arthrosc 2005:13:248-55.

41 Hinman RS, Bennell KL, Metcalf BR, et al. Temporal activity of vastus medialis obliquus and vastus lateralis in symptomatic knee osteoarthritis. Am J Phys Med Rehabil 2002;81:684-90.

42 Hinman RS, Lentzos J, Vicenzino B, et al. Is patellofemoral osteoarthritis common in middle-aged people with chronic patellofemoral pain? Arthritis Care Res 2014:66:1252-7.

43 Huang J, Ushiyama T, Inoue K, et al. Vitamin D receptor gene polymorphisms and osteoarthritis of the hand, hip, and knee: acase-control study in Japan. Rheumatology 2000;39:79-84.

44 Hulet C, Menetrey J, Beaufils P, et al. Clinical and radiographic results of arthroscopic partial lateral meniscectomies in stable knees with a minimum follow up of 20 years. Knee Surg Sports Traumatol Arthrosc 2015;23:225-31.

45 Hunter DJ, Niu J, Zhang Y, et al. Knee height, knee pain, and knee osteoarthritis: the Beijing Osteoarthritis study. Arthritis Rheum 2005;52:1418-23. 
46 Hunter DJ, Zhang YQ, Niu JB, et al. Patella malalignment, pain and patellofemoral progression: the health $A B C$ study. Osteoarthritis Cartilage 2007;15:1120-7.

47 Järvelä T, Paakkala T, Kannus P, et al. The incidence of patellofemoral osteoarthritis and associated findings 7 years after anterior cruciate ligament reconstruction with a bone-patellar tendon-bone autograft. Am J Sports Med 2001;29:18-24.

48 Jones AC, Ledingham J, McAlindon T, et al. Radiographic assessment of patellofemoral osteoarthritis. Ann Rheum Dis 1993;52:655-8.

49 Keays SL, Bullock-Saxton JE, Keays AC, et al. A 6-year follow-up of the effect of graft site on strength, stability, range of motion, function, and joint degeneration after anterior cruciate ligament reconstruction: patellar tendon versus semitendinosus and Gracilis tendon graft. Am J Sports Med 2007;35:729-39.

50 Kerna I, Kisand K, Tamm AE, et al. Two Single-Nucleotide polymorphisms in ADAM12 gene are associated with early and late radiographic knee osteoarthritis in estonian population. Arthritis 2013;2013:1-6.

51 Kujala UM, Kettunen J, Paananen $\mathrm{H}$, et al. Knee osteoarthritis in former runners, soccer players, weight lifters, and shooters. Arthritis Rheum 1995;38:539-46.

52 Kumm J, Tamm A, Lintrop M, et al. The prevalence and progression of radiographic knee osteoarthritis over 6 years in a population-based cohort of middle-aged subjects. Rheumatol Int 2012;32:3545-50.

53 Lacey RJ, Thomas E, Duncan RC, et al. Gender difference in symptomatic radiographic knee osteoarthritis in the knee clinical assessment--CAS(K): a prospective study in the general population. BMC Musculoskelet Disord 2008:9:82.

54 Lanyon P, O'Reilly $\mathrm{S}$, Jones $\mathrm{A}$, et al. Radiographic assessment of symptomatic knee osteoarthritis in the community: definitions and normal joint space. Ann Rheum Dis 1998;57:595-601.

55 Ledingham J, Regan M, Jones A, et al. Radiographic patterns and associations of osteoarthritis of the knee in patients referred to hospital. Ann Rheum Dis 1993;52:520-6.

56 Li RT, Lorenz S, Xu Y, et al. Predictors of radiographic knee osteoarthritis after anterior cruciate ligament reconstruction. Am J Sports Med 2011;39:2595-603

57 Lidén M, Sernert N, Rostgård-Christensen L, et al. Osteoarthritic changes after anterior cruciate ligament reconstruction using bone-patellar tendon-bone or hamstring tendon autografts: a retrospective, 7-year radiographic and clinical followup study. Arthroscopy 2008;24:899-908.

58 Lohmander LS, Ostenberg A, Englund M, et al. High prevalence of knee osteoarthritis, pain, and functional limitations in female soccer players twelve years after anterior cruciate ligament injury. Arthritis Rheum 2004;50:3145-52.

59 McAlindon T, Zhang Y, Hannan M, et al. Are risk factors for patellofemoral and tibiofemoral knee osteoarthritis different? J Rheumatol 1996;23:332-7.

60 McAlindon TE, Snow S, Cooper C, et al. Radiographic patterns of osteoarthritis of the knee joint in the community: the importance of the patellofemoral joint. Ann Rheum Dis 1992;51:844-9.

61 Messier SP, DeVita P, Cowan RE, et al. Do older adults with knee osteoarthritis place greater loads on the knee during gait? A preliminary study. Arch Phys Med Rehabil 2005:86:703-9.

62 Murray JR, Lindh AM, Hogan NA, et al. Does anterior cruciate ligament reconstruction lead to degenerative disease?: thirteen-year results after bonepatellar tendon-bone autograft. Am J Sports Med 2012;40:404-13.

63 Naredo E, Cabero F, Palop MJ, et al. Ultrasonographic findings in knee osteoarthritis: a comparative study with clinical and radiographic assessment. Osteoarthritis Cartilage 2005;13:568-74.

64 Neame R, Zhang W, Deighton C, et al. Distribution of radiographic osteoarthritis between the right and left hands, hips, and knees. Arthritis Rheum 2004; 50:1487-94.

65 Neuman P, Kostogiannis I, Fridén T, et al. Patellofemoral osteoarthritis 15 years after anterior cruciate ligament injury--a prospective cohort study. Osteoarthritis Cartilage 2009;17:284-90

66 Øiestad BE, Holm I, Engebretsen L, et al. The prevalence of patellofemoral osteoarthritis 12 years after anterior cruciate ligament reconstruction. Knee Surgery, Sports Traumatology, Arthroscopy 2013;21:942-9.

67 Roth JH, Kennedy JC, Lockstadt H, et al. Polypropylene braid augmented and nonaugmented intraarticular anterior cruciate ligament reconstruction. Am J Sports Med 1985;13:321-36.

68 Rytter S, Egund N, Jensen LK, et al. Occupational kneeling and radiographic tibiofemoral and patellofemoral osteoarthritis. J Occup Med Toxicol 2009:4:19.

69 Sadat-Ali M, Al-Gindan Y, Al-Mousa M, et al. Osteoarthritis of the knee among saudi arabian security forces personnel. Mil Med 1996;161:105-7.

70 Sajovic M, Vengust $V$, Komadina R, et al. A prospective, randomized comparison of semitendinosus and gracilis tendon versus patellar tendon autografts for anterior cruciate ligament reconstruction: five-year follow-up. Am J Sports Med 2006:34:1933-40

71 Salmon LJ, Russell VJ, Refshauge K, et al. Long-term outcome of endoscopic anterior cruciate ligament reconstruction with patellar tendon autograft: minimum 13-year review. Am J Sports Med 2006;34:721-32.

72 Spector TD, Harris PA, Hart DJ, et al. Risk of osteoarthritis associated with long-term weight-bearing sports: a radiologic survey of the hips and knees in female exathletes and population controls. Arthritis Rheum 1996;39:988-95.
73 Swärd P, Fridén T, Boegård T, et al. Association between varus alignment and posttraumatic osteoarthritis after anterior cruciate ligament injury. Knee Surg Sports Traumatol Arthrosc 2013;21:2040-7

74 Szebenyi B, Hollander AP, Dieppe $\mathrm{P}$, et al. Associations between pain, function, and radiographic features in osteoarthritis of the knee. Arthritis Rheum 2006;54:230-5.

75 Szoeke C, Dennerstein L, Guthrie J, et al. The relationship between prospectively assessed body weight and physical activity and prevalence of radiological knee osteoarthritis in postmenopausal women. J Rheumatol 2006:33:1835-40

76 Tangtrakulwanich B, Geater AF, Chongsuvivatwong V. Prevalence, patterns, and risk factors of knee osteoarthritis in thai monks. J Orthop SCi 2006; 11:439-45.

77 Thorstensson CA, Andersson ML, Jönsson H, et al. Natural course of knee osteoarthritis in middle-aged subjects with knee pain: 12-year follow-up using clinical and radiographic criteria. Ann Rheum Dis 2009:68:1890-3.

78 van der Esch M, Knol DL, Schaffers IC, et al. Osteoarthritis of the knee: multicompartmental or compartmental disease? Rheumatology 2014;53:540-6.

79 Amin S, Baker K, Niu J, et al. Quadriceps strength and the risk of cartilage loss and symptom progression in knee osteoarthritis. Arthritis Rheum 2009;60:189-98.

80 Amin S, Goggins J, Niu J, et al. Occupation-related squatting, kneeling, and heavy lifting and the knee joint: a magnetic resonance imaging-based study in men. J Rheumatol 2008;35:1645-9.

81 Amin S, Guermazi A, Lavalley MP, et al. Complete anterior cruciate ligament tear and the risk for cartilage loss and progression of symptoms in men and women with knee osteoarthritis. Osteoarthritis Cartilage 2008;16:897-902.

82 Cai J, Xu J, Wang K, et al. Association between infrapatellar fat pad volume and knee structural changes in patients with knee osteoarthritis. J Rheumato 2015:42:1878-84.

83 Chan WP, Lang P, Stevens MP, et al. Osteoarthritis of the knee: comparison of radiography, $C T$, and MR imaging to assess extent and severity. AJR Am J Roentgenol 1991;157:799-806.

84 Crema MD, Cibere J, Sayre EC, et al. The relationship between subchondral sclerosis detected with MRI and cartilage loss in a cohort of subjects with knee pain: the knee osteoarthritis progression (KOAP) study. Osteoarthritis Cartilage 2014:22:540-6.

85 Culvenor AG, Collins NJ, Guermazi A, et al. Early knee osteoarthritis is evident one year following anterior cruciate ligament reconstruction. Arthritis Rheumatol 2015;67:946-55

86 Ding C, Cicuttini F, Scott F, et al. Association between age and knee structural change: a cross sectional MRI based study. Ann Rheum Dis 2005;64:549-55.

87 Gross KD, Felson DT, Niu J, et al. Association of flat feet with knee pain and cartilage damage in older adults. Arthritis Care Res 2011:63:937-44.

88 Hayes CW, Jamadar DA, Welch GW, et al. Osteoarthritis of the knee: comparison of MR imaging findings with radiographic severity measurements and pain in middleaged women. Radiology 2005;237:998-1007.

89 Kornaat PR, Watt I, Riyazi N, et al. The relationship between the MRI features of mild osteoarthritis in the patellofemoral and tibiofemoral compartments of the knee. Eur Radiol 2005:15:1538-43.

90 Peterfy CG, Guermazi A, Zaim S, et al. Whole-organ magnetic resonance imaging score (WORMS) of the knee in osteoarthritis. Osteoarthritis Cartilage 2004;12:177-90.

91 Runhaar J, Schiphof D, van Meer B, et al. How to define subregional osteoarthritis progression using semi-quantitative MRI Osteoarthritis Knee Score (MOAKS). Osteoarthritis Cartilage 2014;22:1533-6.

92 Sharma L, Chmiel JS, Almagor O, et al. Significance of preradiographic magnetic resonance imaging lesions in persons at increased risk of knee osteoarthritis. Arthritis Rheumatol 2014;66:1811-9.

93 Sowers M, Karvonen-Gutierrez CA, Jacobson JA, et al. Associations of anatomical measures from MRI with radiographically defined knee osteoarthritis score, pain, and physical functioning. J Bone Joint Surg Am 2011;93:241-51.

94 Stefanik JJ, Gross KD, Guermazi A, et al. Relation of step length to magnetic resonance Imaging-Detected structural damage in the patellofemoral joint: the multicenter osteoarthritis study. Arthritis Care Res 2016:68:776-83.

95 Stefanik JJ, Niu J, Gross KD, et al. Using magnetic resonance imaging to determine the compartmental prevalence of knee joint structural damage. Osteoarthritis Cartilage 2013:21:695-9.

96 Teng HL, MacLeod TD, Link TM, et al. Higher knee flexion moment during the second half of the stance phase of gait is associated with the progression of osteoarthritis of the patellofemoral joint on magnetic resonance imaging. J Orthop Sports Phys Ther 2015:45:656-64.

97 Tsavalas N, Katonis P, Karantanas AH. Knee joint anterior malalignment and patellofemoral osteoarthritis: an MRI study. Eur Radio/ 2012;22:418-28.

98 van Meer BL, Oei EH, Meuffels DE, et al. Degenerative changes in the knee 2 years after anterior cruciate ligament rupture and related risk factors: a prospective observational Follow-up study. Am J Sports Med 2016;44:1524-33.

99 Wang J, Antony B, Zhu Z, et al. Association of patellar bone marrow lesions with knee pain, patellar cartilage defect and patellar cartilage volume loss in older adults: a cohort study. Osteoarthritis Cartilage 2015;23:1330-6. 
100 Wang Y, Dempsey AR, Lloyd DG, et al. Patellofemoral and tibiofemoral articular cartilage and subchondral bone health following arthroscopic partial medial meniscectomy. Knee Surg Sports Traumatol Arthrosc 2012;20:970-8.

101 Stefanik JJ, Gross KD, Guermazi A, et al. The relation of MRI-detected structural damage in the medial and lateral patellofemoral joint to knee pain: the multicenter and Framingham osteoarthritis studies. Osteoarthritis Cartilage 2015;23:565-70.

102 Crossley KM, Vicenzino B, Lentzos J, et al. Exercise, education, manual-therapy and taping compared to education for patellofemoral osteoarthritis: a blinded, randomised clinical trial. Osteoarthritis Cartilage 2015;23:1457-64.

103 Callaghan MJ, Parkes MJ, Hutchinson CE, et al. A randomised trial of a brace for patellofemoral osteoarthritis targeting knee pain and bone marrow lesions. Ann Rheum Dis 2015:74:1164-70.

104 Hunter DJ, Harvey W, Gross KD, et al. A randomized trial of patellofemoral bracing for treatment of patellofemoral osteoarthritis. Osteoarthritis Cartilage 2011;19:792-800.
105 Utting MR, Davies G, Newman JH. Is anterior knee pain a predisposing factor to patellofemoral osteoarthritis? Knee 2005;12:362-5.

106 Thomas MJ, Wood L, Selfe J, et al. Anterior knee pain in younger adults as a precursor to subsequent patellofemoral osteoarthritis: a systematic review. BMC Musculoskelet Disord 2010;11:201.

107 Roemer FW, Kwoh CK, Hannon MJ, et al. What comes first? multitissue involvement leading to radiographic osteoarthritis: magnetic resonance Imaging-Based trajectory analysis over four years in the osteoarthritis initiative. Arthritis Rheumatol 2015;67:2085-96.

108 Javaid MK, Kiran A, Guermazi A, et al. Individual MRI and radiographic features of knee $\mathrm{OA}$ in subjects with unilateral knee pain: health $\mathrm{ABC}$ study. Arthritis Rheum 2012;64:3246-55.

109 Pelletier JP, Cooper C, Peterfy C, et al. What is the predictive value of MRI for the occurrence of knee replacement surgery in knee osteoarthritis? Ann Rheum Dis 2013;72:1594-604. 